\title{
Generalisability of a Virtual Trials Method for Glycaemic Control in Intensive Care
}

\author{
Jennifer L. Dickson, Kent. W. Stewart, Christopher G. Pretty, Member, IEEE, Marine Flechet, \\ Thomas Desaive, Sophie Penning, Bernard C. Lambermont, Balázs Benyó, Member, IEEE, Geoffrey \\ M. Shaw, J. Geoffrey Chase
}

\begin{abstract}
Background: Elevated blood glucose (BG) concentrations (Hyperglycaemia) are a common complication in critically ill patients. Insulin therapy is commonly used to treat hyperglycaemia, but metabolic variability often results in poor BG control and low BG (hypoglycaemia). Objective: This paper presents a modelbased virtual trial method for glycaemic control protocol design, and evaluates its generalisability across different populations. Methods: Model-based insulin sensitivity (SI) was used to create virtual patients from clinical data from three different ICUs in New Zealand, Hungary, and Belgium. Glycaemic results from simulation of virtual patients under their original protocol (self-simulation) and protocols from other units (cross-simulation) were compared. Results: Differences were found between the three cohorts in median SI and inter-patient variability in SI. However, hour-to-hour intra-patient variability in SI was found to be consistent between cohorts. Self and crosssimulation results were found to have overall similarity and consistency, though results may differ in the first 24-48 hours due to different cohort starting BG and underlying SI. Conclusions and significance: Virtual patients and the virtual trial method were found to be generalisable across different ICUs. This virtual trial method is useful for in silico protocol design and testing, given an understanding of the underlying assumptions and limitations of this method.

Index Terms-Glycaemic control, virtual trials, insulin sensitivity
\end{abstract}

\section{INTRODUCTION}

C ritically ill patients often experience sustained stress sinduced hyperglycaemia (high blood sugar) and high glycaemic variability due to their critical condition [1-4]. Hypoglycaemia (low blood sugar), hyperglycaemia and high

Paper submitted 5 August 2016. Corresponding author: J. L. Dickson. J. L. Dickson, C.G Pretty, K. W. Stewart, and J. G. Chase are with the Department of Mechanical Engineering, University of Canterbury, Christchurch, New Zealand (e-mail: jennifer.dickson@canterbury.ac.nz,

chris.pretty@canterbury.ac.nz, kent.stewart@pg.canterbury.ac.nz, geoff.chase@canterbury.ac.nz).

T. Desaive, M. Flechet, and S. Penning, did this research as part of the GIGA group, Department of Thermodynamics of Irreversible Processes, University of Liege, Liege, Belgium (email: marine.flechet@kuleuven.be, tdesaive@ulg.ac.be, sophie.penning@gmail.com).

B. C. Lambermont is with the University Hospital of Liege, Liege, Belgium (email: b.lambermont@chu.ulg.ac.be). glycaemic variability have each been independently linked to increased morbidity and mortality in intensive care units (ICU) [5-11]. Glycaemic control has demonstrated the ability to improve blood glucose (BG) outcomes, and reduce morbidity and mortality [12-15], based on the control of glycaemic levels [10, 16-20]. However, several studies have failed to repeat these positive results [21-23], often with significantly increased hypoglycaemia [11]. The main issue is that ICU patients are highly variable in their response to insulin, particularly in the first 48 hours of stay [24-26], which can make managing glycaemia difficult as patient condition evolves [27].

In this regard, model-based glycaemic control methods have recently shown good safety and performance [28-33]. Their main advantage is the use of a computer model to identify patient-specific insulin sensitivity (SI), which quantitatively describes patient response to insulin. In this way model-based protocols can better account for inter- and intra- patient variability when dosing insulin, in ways that many non-modelbased protocols simply cannot. However, given a validated model, these protocols are only as good as the ability to design the protocol for safe, effective glycaemic control.

Assessing glycaemic control protocols in silico using clinically validated models and virtual trial methods enables the ability to design new protocols or assess and improve existing protocols, to optimize safety and quality of glycaemic control. Virtual trials utilise SI to simulate per-patient BG response to protocolised insulin treatments, and thus allow the assessment of hypo- and hyper- glycaemic risks and glycaemic variability. In silico design is also much faster and more efficient than controlled clinical testing on patients, with far less patient risk. Thus, a protocol can be optimized safely and rapidly before validation clinical pilot tests on patients. Importantly, this approach usually requires local clinical data to create virtual patients, characterised by time-varying insulin sensitivity, on

B. Benyó is with the Department of Control Engineering and Information Technology, Budapest University of Technology and Economics, Budapest, Hungary (email: bbenyo@iit.bme.hu).

Geoffrey M. Shaw is with the Department of Intensive Care, Christchurch Hospital, and also the University of Otago, Christchurch, New Zealand (email: geoff.shaw@cdhb.govt.nz).

The authors acknowledge the support of the EU FP7 and RSNZ Marie Curie IRSES fund, and the University of Canterbury Doctoral Scholarship Scholarship.supporting KS, the Belgian FNRS for support of SP, and the NZ MedTech CoRE for partial support of JLD. 
which to test these protocols [34-36]. Without this data, it is not possible to be sure if a protocol will, even with good compliance, translate successfully to another ICU.

The objective of this paper is to assess the potential of independent (non-local) cohorts to generate virtual patients and simulate outcomes of glycaemic control protocols. This will be done in two parts:

1) Analysis of model-based insulin sensitivity (SI) and its variability in three different ICU cohorts.

2) Use of SI to create virtual patients for simulation, both with the original protocol (Self-Simulation) and protocols from other cohorts (Cross-Simulation), where results are compared to the clinical data for that protocol and cohort. The first examines underlying metabolic similarities/differences between the cohorts, and the second assesses the impact of these similarities and differences in assessing glycaemic control outcomes in virtual patient simulation. Hence, these two objectives test the interchangeability of virtual patients for virtual trial testing across units, cohorts and protocols, where the greater the generality, the easier it is to test and design new solutions using virtual patients.

\section{Patient data, Cohorts, And Protocols}

Retrospective clinical data from three independent cohorts of 20 critically ill patients were used for this study from 3 mixedmedical ICU's in Belgium, Hungary, and New Zealand. Patients were selected from clinical data records in each ICU based on similar BG data density required to create the equivalent, good quality virtual patients [37]. This criterion ensures more consistent measurement of true similarity or differences of metabolic differences between cohorts [38, 39].
Diagnosed diabetic patients were excluded. Other selection criteria for patients were: (1) glycaemic control for at least 60 hours; (2) insulin administration at the beginning of glycaemic control; (3) at least 10 BG measurements during control, every 4 hours (on average, but preferably more frequent), to allow good virtual patients to be created. Clinical data only covers time when insulin was being administered regularly, and insulin weaning protocols differed between units. Typically insulin therapy is completed well before ICU discharge. Clinical data was limited to a maximum of 120 hours ( 5 days) per patient, to prevent data from the more variable first 24 hours being swamped by more stable long glycaemic control episodes. This also prevented a few longer stay patients from dominating the outcome statistics. The total hours per cohort in Table 1 are approximately the 2000-2500 hours required to quantify metabolic level and variability in [38].

All three cohorts are treated differently, and they are not intentionally clinically matched by APACHE code, diagnosis or other metrics (this data was not available for the Belgian cohort), although age is similar. Stage one of the analysis will assess metabolic similarity of these three cohorts. Thus, these three cohorts will provide a robust test of the hypothesis of interchangeability across different mixed-medical ICU's and countries. Cohort details are shown in Table 1 , and from here forward, the cohorts are denoted: Hungary (HU), New Zealand (NZ) and Belgium (BE). Although insulin delivery rates are similar between cohorts, nutritional dextrose delivery is very different (Table 1, Figure 1). Between the NZ and HU cohorts, APACHE II scores are very different. This data was not available for the BE cohort.

\section{A. Belgium (BE)}

The first cohort is from the Centre Hospitalier Universitaire

TABLE I

COHORT DETAILS. DATA ARE SHOWN AS MEDIAN[IQR] WHERE APPROPRIATE.

\begin{tabular}{|l|c|c|c|}
\hline \hline Parameters & New Zealand (NZ) & Hungarian (HU) & Belgian (BE) \\
\hline Num patients & 20 & 20 & 20 \\
\hline Total hours & 1915 & 2000 & 2222 \\
\hline Age (years) & $65.0[54.8-68.5]$ & $66.5[53.8-70.0]$ & $66.0[51.8-71.8]$ \\
\hline Gender (M/F) & $14 / 6$ & $14 / 6$ & $9 / 11$ \\
\hline APACHE II Score [IQR] & $24[19-28] *$ & $33[29-37] *$ & N/A \\
\hline ICU LoS [IQR] (days) & $20.0[7.2-35.7]^{*}$ & $14.0[8.2-19.8] * *$ & N/A \\
\hline Time on Protocol (h) & $139.2[82.8-216.8]$ & $111.0[77.1-192.1]$ & $196.9[114.9-291.0]$ \\
\hline Starting BG [mmol/L] & $10.8[9.0-13.9]$ & $8.2[6.9-9.6]$ & $8.5[7.3-9.9]$ \\
\hline Diabetic & None & None & None \\
\hline Insulin dose (U/hr) & $3.0[1.5-4.5]$ & $3.0[1.5-5.0]$ & $3.0[2.0-5.5]$ \\
\hline Glucose Intake (g/hr) & $6.1[4.0-6.7]$ & $8.2[6.0-10.6]$ & $9.7[8.3-11.3]$ \\
\hline Model based SI: (L/mU/min $x$ 10-4) & & & \\
\hline Median SI [IQR] & $3.2[2.4-4.4]$ & $4.8[3.5-6.7]$ & $2.0[0.8-4.2]$ \\
\hline Median hour-to-hour $\Delta$ SI [IQR] & $0.04[-0.3-+0.4]$ & $0.05[-0.4-+0.4]$ & $0.01[-0.2-+0.2]$ \\
\hline Median absolute hour-to-hour $\Delta$ SI [IQR] & $0.3[0.1-0.7]$ & $0.4[0.2-0.8]$ & $0.2[0.1-0.4]$ \\
\hline Median [IQR] Per-patient Median SI & $3.2[2.8-3.7]$ & $5.1[3.8-5.7]$ & $3.9[3.1-4.9]$ \\
\hline Model \% fitting error [IQR] & $-0.6[-2.2-0.8]$ & $0.1[-1.3-1.5]$ & $0.0[-1.1-1.0]$ \\
\hline \hline
\end{tabular}

*This data was available for 17/20 patients. **This data was available for 15/20 patients. N/A - this data was not available for the BE cohort. 

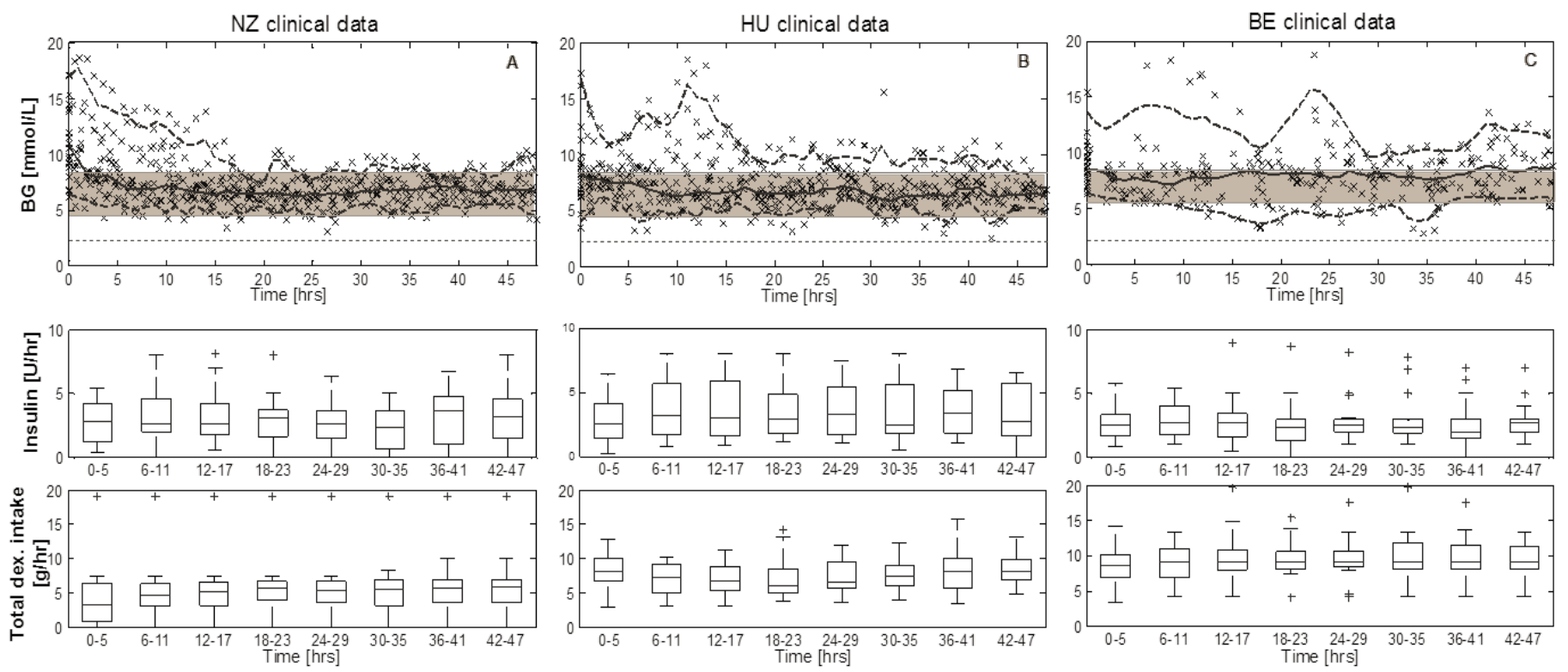

Fig. 1. First 24 hours of clinical data for a) NZ cohort, b) HU cohort, and c) BE cohort. Dashed lines show the $5^{\text {th }}$ and $95^{\text {th }}$ percentiles of BG over time, and the lowermost dotted line denotes the formal definition for hypoglycemic threshold $(2.2 \mathrm{mmol} / \mathrm{L})$. Average insulin dose and total dextrose (parenteral and enteral) intake is shown in 6 hour blocks.

of Liège (CHU) ICU, Belgium. In this cohort, the standard of care glycaemic control protocol was a sliding scale that dosed insulin based on the patient's BG level and the previous insulin dose [40]. The protocol applied at CHU of Liège targets the 5.6$8.3 \mathrm{mmol} / \mathrm{L}(100-150 \mathrm{mg} / \mathrm{dL})$ band, and is characterized by an insulin infusion-only approach with a 1- or 4- hour time interval between BG measurements. Insulin rate is adjusted depending on current and previous BG level and current insulin infusion rate. However, it is limited by an inability to reduce insulin infusions until BG is within the target range. Enteral and parenteral nutrition is decided by clinicians and ICU practice with glucose boluses specified for $\mathrm{BG}<2.2 \mathrm{mmol} / \mathrm{L}$. BG measurements are made hourly until BG values are stable in the band, and then 4-hourly. Full details are given in [40].

\section{B. New Zealand (NZ)}

The second cohort is from the Christchurch Hospital ICU, New Zealand, which uses the stochastic model-based STAR glycaemic control protocol [32, 33, 41]. The STAR protocols adjust both insulin and nutrition levels $[32,41]$ and measures hourly when outside the 4.4-8.0 mmol/L (80-144 mg/dL) band, and up to 3-hourly within the band based on nursing choice of intervention interval. The goal is to control BG with a $5 \%$ risk of $\mathrm{BG}<4.4 \mathrm{mmol} / \mathrm{L}(80 \mathrm{mg} / \mathrm{dL})$ for each intervention and to otherwise maximize nutrition given towards a target of 25 $\mathrm{kcal} / \mathrm{kg} /$ day, as per ACCP and ESPEN guidelines [42, 43]. Insulin is typically delivered as boluses every hour via an infusion pump.

STAR utilizes stochastic forecasting of likely BG outcomes based on models of metabolic variability observed across a representative clinical data set. Hour-to-hour metabolic variability is characterized by a model-based insulin sensitivity (SI) parameter, and models of variability in SI allow subsequent forecasting of likely BG outcomes for the next 1,2 , and 3 hours [38]. These models can be used to dose insulin based on risk of glycaemic outcomes, and in particular limiting the risk of $\mathrm{BG}<4.4 \mathrm{mmol} / \mathrm{L}$ to $5 \%$ or less [32, 41]. The stochastic SI forecasting model used by this protocol is based on a retrospective reference population [38]. Insulin is automatically stopped by the STAR protocol when high insulin sensitivity indicates exogenous insulin is no longer required. Complete details on the STAR control framework can be found elsewhere [32, 41].

\section{Hungary (HU)}

The third cohort is from Kálmán Pándy Hospital ICU, Hungary, which also uses STAR, although differently within the same framework. In the Hungarian cohort a STARframework (STARHu) delivers insulin as an intravenous infusion and uses a significantly higher carbohydrate nutrition formula than the New Zealand ICU. Patients are transitioned from early aggressive parenteral nutrition to enteral nutrition as their stay progresses. Hence, it has a similar $5 \%$ risk of $B G<4.4$ $\mathrm{mmol} / \mathrm{L}$, but delivers insulin and nutrition very differently. The stochastic model of SI used was the same as that used in NZ. Insulin is automatically stopped by the STAR protocol when high insulin sensitivity indicates exogenous insulin is no longer required.

\section{Physiological Model and Virtual Patients}

The model used to create virtual patients is the clinically validated intensive care insulin, nutrition and glucose (ICING) model [44-46]:

$$
\begin{array}{r}
\dot{G}(t)=-p_{G} G(t)-S_{I}(t) G(t) \frac{Q(t)}{1+\alpha_{G} Q(t)} \\
+\frac{P(t)+E G P-C N S}{V_{G}}
\end{array}
$$




$$
\begin{gathered}
\dot{Q}(t)=n_{I}(I(t)-Q(t))-n_{C} \frac{Q(t)}{1+\alpha_{G} Q(t)} \\
\dot{I}(t)=-n_{K} I(t)-n_{L} \frac{I(t)}{1+\alpha_{I} I(t)}-n_{I}(I(t)-Q(t)) \\
+\frac{u_{e x}(t)}{V_{I}}+\left(1-x_{L}\right) \frac{u_{e n}(G)}{V_{I}} \\
P(t)=\min \left(d_{2} P_{2}, P_{\max }\right)+P_{P N}(t) \\
\dot{P}_{1}(t)=-d_{1} P_{1}+P_{E N}(t) \\
\dot{P}_{2}(t)=-\min \left(d_{2} P_{2}, P_{\max }\right)+d_{1} P_{1} \\
u_{e n}(G)=\min \left(\max \left(u_{\min }, k_{1} G(t)+k_{2}\right), u_{\max }\right)
\end{gathered}
$$

The key variables and model parameters are described in Table 2. With the exception of model-based insulin sensitivity, $S_{I}(t)$ (SI), all parameters are fixed and have been previously identified from other studies and data cohorts [44, 46, 47], with details in an online appendix to this paper.

This model-based insulin sensitivity, $S_{I}(t)(\mathrm{SI})$, is identified hourly, using the integral based fitting method [48]. SI is representative of 'whole body' underlying metabolic condition, and captures patient-specific deviation from model population parameters. Previous work has shown it to be relatively independent of both insulin and nutrition inputs [35]. It can thus be used to calculate the likely BG response to treatments other than those given clinically. This process is called a virtual trial, and has been used to design protocols for use in clinical situations [35, 49].

To generate virtual patients, clinical data, comprising BG measurements and insulin and nutrition inputs, is used with model Equations (1)-(7) to identify a model-based $S_{I}(t)$ profile for each patient using integral-based methods [48, 50]. These profiles can be used to simulate the response to a new or modified set of insulin and/or nutrition inputs, generating a new G(t) (BG) profile. This virtual patient and virtual trial process is illustrated in Figure 2.

\section{ANALYSES}

\section{A. Comparison of SI}

Model-identified SI values are presented to assess the metabolic similarity of the cohorts. Cumulative distributions are used to assess cohort SI and cohort-wide variability, where offset between curves shows difference in median SI, and the slope denotes the tightness of the distribution. At a given cumulative fraction, $\mathrm{F}$, the results show the median, interquartile range, and $90^{\text {th }}$ percentile range in SI for the per-patient $(100 x F)^{\text {th }}$ percentile SI values. This is a measure of inter-patient variability within each cohort.

Hour-to-hour SI variability is also assessed, using hour-to-

\begin{tabular}{|c|c|c|}
\hline & UNITS & DESCRIPTION \\
\hline \multicolumn{3}{|c|}{ Physiological states } \\
\hline$G(t)$ & $\mathrm{mmol} / \mathrm{l}$ & Blood glucose concentration \\
\hline$I(t)$ & $\mathrm{mU} / \mathrm{l}$ & Plasma insulin concentration \\
\hline$Q(t)$ & $\mathrm{mU} / \mathrm{l}$ & Interstitial insulin concentration \\
\hline$P_{1}(t)$ & mmol & Glucose in stomach \\
\hline$P_{2}(t)$ & mmol & Glucose in gut \\
\hline \multicolumn{3}{|c|}{ External inputs } \\
\hline$P_{P N}(t)$ & $\mathrm{mmol} / \mathrm{min}$ & Parenteral glucose intake \\
\hline$P_{E N}(t)$ & $\mathrm{mmol} / \mathrm{min}$ & Enteral glucose appearance \\
\hline$u_{e x}(t)$ & $\mathrm{mmol} / \mathrm{min}$ & Exogenous Insulin administration \\
\hline \multicolumn{3}{|c|}{ Model parameters } \\
\hline$S_{I}(t)$ & $\mathrm{l} / \mathrm{mU} / \mathrm{min}$ & Insulin sensitivity \\
\hline$\alpha_{G}$ & $1 / 65 \mathrm{l} / \mathrm{mU}$ & $\begin{array}{l}\text { Saturation of insulin-mediated glucose } \\
\text { uptake }\end{array}$ \\
\hline$V_{\mathrm{G}}$ & $13.3 \mathrm{~L}$ & Glucose distribution volume \\
\hline$E G P$ & $1.16 \mathrm{mmol} / \mathrm{min}$ & $\begin{array}{l}\text { Endogenous glucose production } \\
\text { (hepatic) }\end{array}$ \\
\hline CNS & $0.3 \mathrm{mmol} / \mathrm{min}$ & $\begin{array}{l}\text { Glucose uptake by central nervous } \\
\text { system }\end{array}$ \\
\hline$d_{1}$ & $-\ln (0.5) / 20 \mathrm{~min}^{-1}$ & $\begin{array}{l}\text { Rate parameter: gastric emptying of } \\
\text { stomach to gut }\end{array}$ \\
\hline$d_{2}$ & $-\ln (0.5) / 100 \min ^{-1}$ & $\begin{array}{l}\text { Rate parameter: glucose absorption for } \\
\text { gut to bloodstream }\end{array}$ \\
\hline$P_{\max }$ & $6.11 \mathrm{mmol} / \mathrm{min}$ & $\begin{array}{l}\text { Maximum glucose absorption rate from } \\
\text { gut }\end{array}$ \\
\hline$x_{L}$ & 0.67 & $\begin{array}{l}\text { Fractional first pass hepatics insulin } \\
\text { clearance from portal vein }\end{array}$ \\
\hline$n_{L}$ & $0.1578 \mathrm{~min}^{-1}$ & $\begin{array}{l}\text { Rate parameter: general hepatic insulin } \\
\text { clearance }\end{array}$ \\
\hline$\alpha_{I}$ & $1.7 \times 10-3 \mathrm{l} / \mathrm{mU}$ & Saturation of hepatics insulin clearance \\
\hline$n_{K}$ & $0.0542 \mathrm{~min}^{-1}$ & $\begin{array}{l}\text { Rate parameter: kidney clearance of } \\
\text { insulin }\end{array}$ \\
\hline$n_{C}$ & $0.0075 \mathrm{~min}^{-1}$ & $\begin{array}{l}\text { Rate parameter: cellular degradation of } \\
\text { internalised insulin }\end{array}$ \\
\hline$n_{I}$ & $0.0075 \mathrm{~min}^{-1}$ & $\begin{array}{l}\text { Rate parameter: diffusion of insulin } \\
\text { between plasma and interstitium }\end{array}$ \\
\hline$u_{e n}$ & $\mathrm{mU} / \mathrm{min}$ & Pancreatic insulin secretion \\
\hline$k_{1}$ & $\begin{array}{c}14.9 \\
\mathrm{mU} \cdot \mathrm{l} / \mathrm{mmol} / \mathrm{min}\end{array}$ & Insulin secretion model parameter \\
\hline$k_{2}$ & $-49.9 \mathrm{mU} / \mathrm{min}$ & Insulin secretion model parameter \\
\hline$u_{\min }$ & $16.7 \mathrm{mU} / \mathrm{min}$ & Minimum insulin secretion \\
\hline$u_{\max }$ & $266.7 \mathrm{mU} / \mathrm{min}$ & Maximum insulin secretion \\
\hline$V_{\mathrm{I}}$ & $4.0 \mathrm{~L}$ & Insulin distribution volume \\
\hline
\end{tabular}
hour stochastic models [38, 39], which are plotted with the $x$ axis showing SI at time $\mathrm{n}$ and the $\mathrm{y}$ axis showing SI at time $\mathrm{n}+$ 1. These stochastic hour-to-hour variability plots give a measure of intra-patient metabolic variability [38, 39], which
TABLE II

KEY VARIABLES OF THE ICING METABOLIC GLUCOSE MODEL

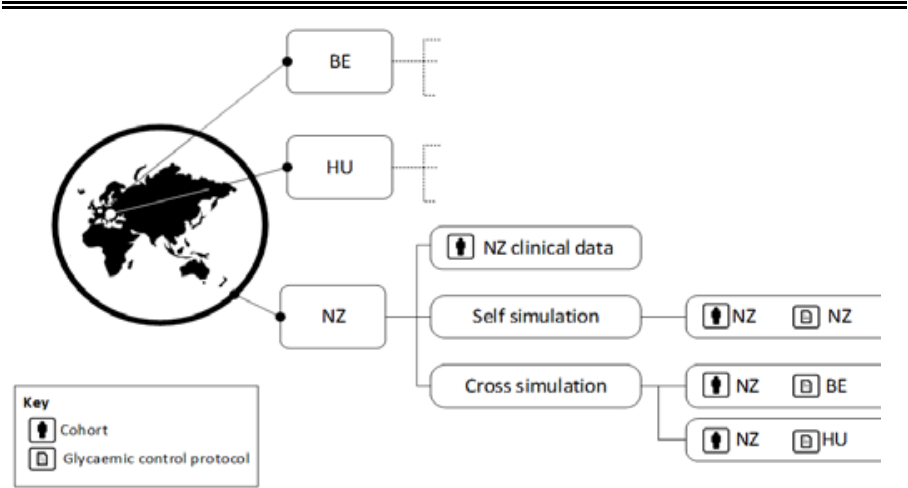

Fig. 2. Virtual trial scheme for self- and cross simulation for the three cohorts and protocols. The full simulation scheme is shown for the NZ cohort only, but generalises to the $\mathrm{BE}$ and $\mathrm{HU}$ cohorts. Self-simulation is used to indicate ability to replicate clinical data. Cross simulation results indicate generalisability of virtual trial methods. 

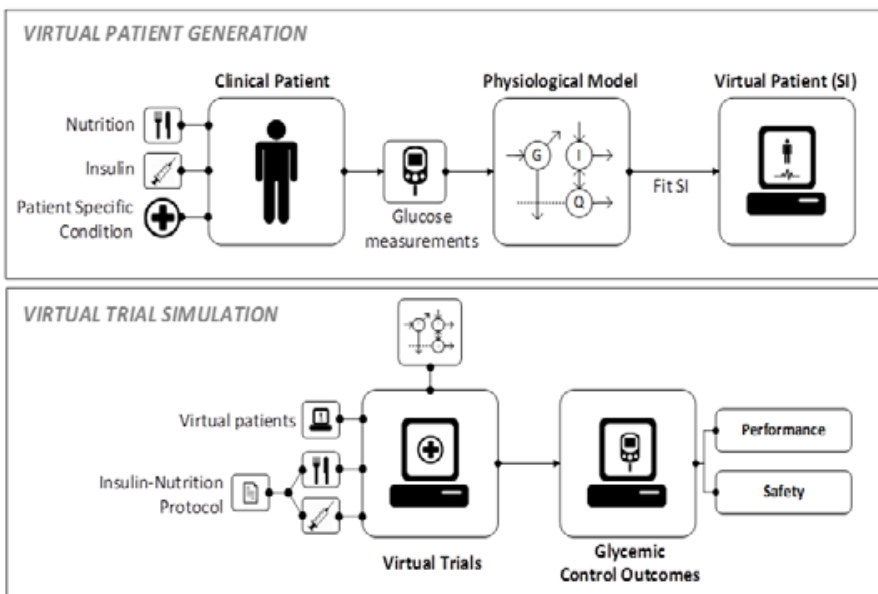

Fig. 3. Virtual patient generation and virtual trial methodology. Virtual patients are comprised of the insulin sensitivity (SI) traces fitted from clinical data Different glycaemic control protocols can be tested in silico to assess likely blood glucose outcomes in individual patients and across a cohort.

can be a leading cause of poor control and hypoglycaemia [27]. If metabolic level and/or variability are similar between cohorts then the SI levels and/or stochastic models will be similar or identical. Consistency between ICUs in SI behaviour would imply that a perfectly matched cohort or data from a specific ICU is not necessary to obtain valid virtual trial results for another ICU, and a small, 20-patient virtual cohort could generalize and capture the metabolic behaviour of a similar mixed-medical ICU.

\section{B. Virtual Trial Simulation}

To examine the relationship between virtual patients and glycaemic control outcomes virtual trials are used. Glycaemic outcomes are a function of SI and its variability, as well as the insulin-nutrition protocol used. If virtual patients are generalisable, glycaemic virtual trial control outcome results will be consistent for all three cohorts (HU, NZ, BE) on that protocol. The results are presented as cumulative distribution functions (CDFs) of BG values. This virtual trial simulation structure is shown in Figure 3.

Testing a virtual patient cohort on the same protocol that yielded the data used to create the virtual patient (selfsimulation) should return exactly the clinical BG data used to generate it, with differences due to model error (also observable as fitting error) and/or lack of compliance in following the protocol clinically. Thus, self-simulation captures the model error and any un-modelled non-compliance to the clinical protocol.

Cross-simulation is a technique for assessing how results of an analysis will generalize to a separate, independent virtual patient cohort. In this case, this step consists in applying a clinical protocol to a series of virtual patients created from a cohort on which the protocol was never clinically tested. If patients are matched in terms of metabolic dynamics and if the virtual trial method is correct, then the in silico results and clinical data would theoretically match or be very similar, showing the generalisability or robustness of the approach. Differences would be due to underlying metabolic differences, and the interaction of model-based SI with the protocol.

Each of the three protocols, where STAR in Hungary and New Zealand are denoted STAR-Hu and STAR-NZ respectively, was simulated on virtual patients from all other cohorts (HU, NZ, BE). In all cases, the virtual trial simulation was only allowed to modulate insulin, as the BE glycaemic
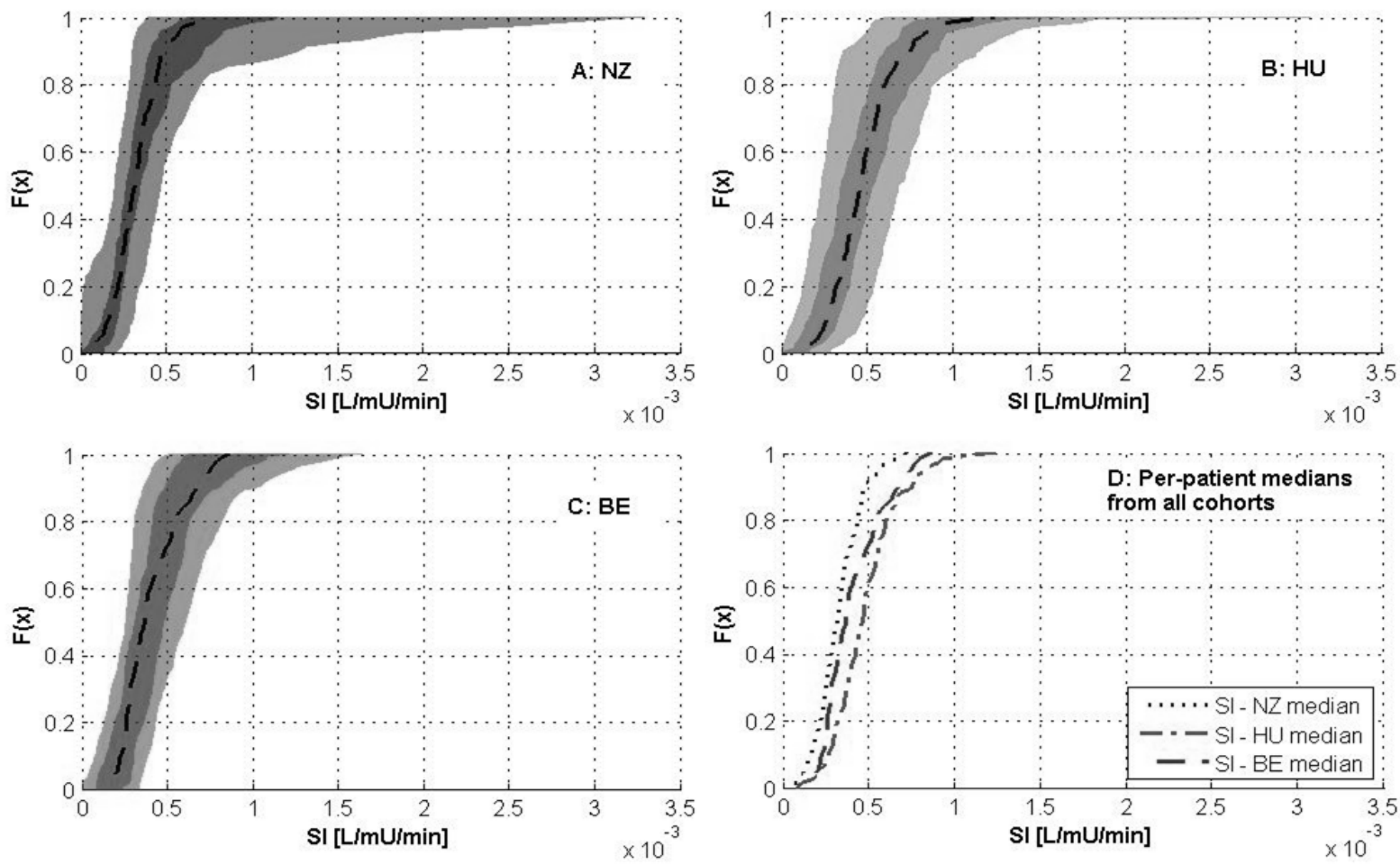

Fig. 4. Per patient median insulin sensitivity (SI) CDFs, for each cohort: a) NZ cohort; b) HU cohort; c) BE cohort; and d) the median per-patient SI CDFs overlaid for comparison. All values identified from data and the ICING model of Equations (1)-(7). In a) $-c)$ the $5^{\text {th }}-95^{\text {th }}$ and $25^{\text {th }}-75^{\text {th }}$ percentile ranges are shown. $\mathrm{F}(\mathrm{x})$ is the cumulative fraction of data less than or equal to the corresponding SI, as per standard CDF definition. 

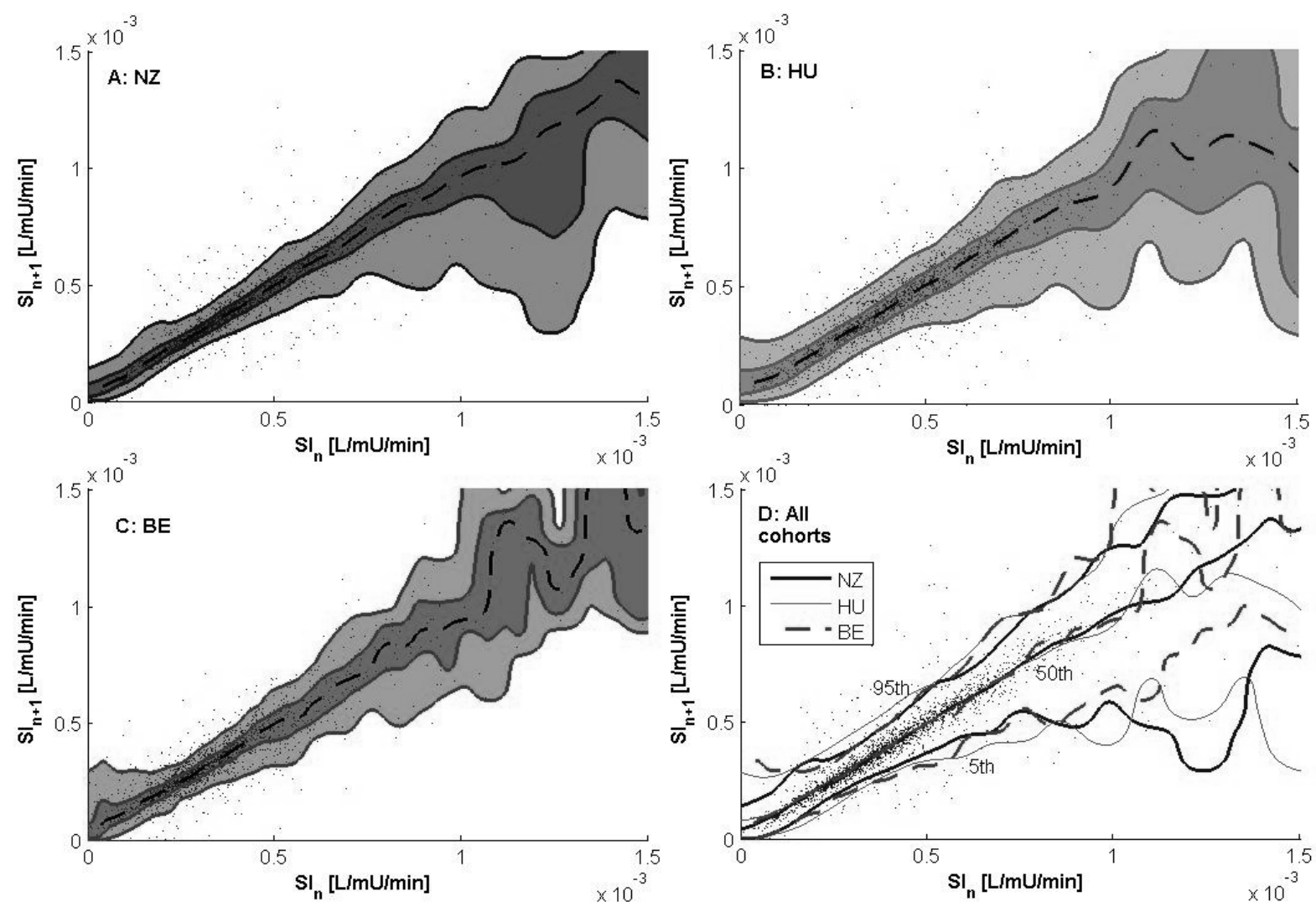

Fig. 5. Stochastic insulin sensitivity models for hour " $n$ ” (x-axis) and hour " $n+1$ ” (y-axis) showing the data (dots) and the 5th-95th percentile range (light), IQR (dark) and median (dashed) probability bounds for stochastic models for the three cohorts, where: a) NZ cohort; b) HU cohort; c) BE cohort; and d) All three cohorts overlaid with the median, $5^{\text {th }}$, and $95^{\text {th }}$ percentile lines shown. All data were found using the ICING model of Equations (1)-(7) to identify hourly SI values.

control protocol did not explicitly modify or determine nutritional intake. In simulation nutrition was set at what was clinically given.

\section{RESULTS}

\section{A. Metabolic Similarity: Inter-Cohort Insulin Sensitivity and Variability}

To examine underlying metabolic similarities and differences between the BE, HU, and NZ cohorts, model-based SI is examined. Whole cohort and per-patient median SI is given in Table 1, along with the median hour-to-hour change in SI, and the median absolute hour-to-hour change in SI. The median hour-to-hour change in SI is $\sim 0$ for each cohort, while the median absolute hour-to-hour change, reflecting both increase and decrease in SI, in SI is approximately $5-10 \%$ of the median SI value.

Figure 4 shows the range of per-patient CDFs SI for each cohort with the median SI overlaid. Median SI is different within each cohort, indicating different degrees of underlying metabolic response to glucose and insulin treatments.

Steeper CDFs indicate lower cohort wide variability as a greater proportion of time is spent around a particular SI range. The consistent slope of the CDFs in Figure 4d shows each cohort is similar in the tightness of the middle $\sim 60 \%$ of the data. However, in Figure 4c the NZ cohort has slightly lower interpatient variability compared to the others, as seen in the lower spread of the $25^{\text {th }}-75^{\text {th }}$ and $5^{\text {th }}-95^{\text {th }}$ percentile SI CDFs. In contrast, the HU cohort has the widest set of per-patient SI CDFs and thus the greatest inter-patient variability. Overall, the magnitude of the model-based SI, and thus the metabolic ability to remove glucose from the blood, is different for each cohort, as seen when comparing medians and offset from other CDFs. SI is different within each cohort, and while cohort wide variability is similar, the degree of inter-patient variability differs between cohorts.

Figure 5 shows the hour-to-hour change in insulin sensitivity

TABLE III

NEW ZEALAND PROTOCOL: CROSS SIMULATION RESULTS, CLINICAL DATA SHADED. RESULTS ARE FOR THE FULL (MAX 120 HRS) PATIENT EPISODE

\begin{tabular}{cccc}
\hline \hline New Zealand (NZ) protocol & NZ - Clinical data & $\begin{array}{c}\text { Cross validation on } \\
\text { HU cohort }\end{array}$ & $\begin{array}{c}\text { Cross validation on } \\
\text { BE cohort }\end{array}$ \\
\hline BG measures / day & 13.1 & 12.9 & 14.2 \\
Mean time between measurements (hr) & $1.8[1.0-2.8]$ & $2.0[1.0-3.0]$ & $1.0[1.0-2.0]$ \\
Median BG [IQR] (mmol/L) & $6.7[6.0-7.3]$ & $6.7[6.2-7.4]$ & $6.9[6.3-7.6]$ \\
Time in 4.4-8.0 mmol/L band (\%) & 85.4 & 83.1 & 81.6 \\
Median [IQR] exogenous insulin bolus (U/hr) & $3.0[1.5-4.5]$ & $2.5[1.5-4.0]$ & $4.0[2.0-6.0]$ \\
\hline Per-Patient Median BG (mmol/L) & $6.6[6.4-6.8]$ & $6.7[6.5-6.9]$ & $6.9[6.6-7.1]$ \\
\hline \hline
\end{tabular}



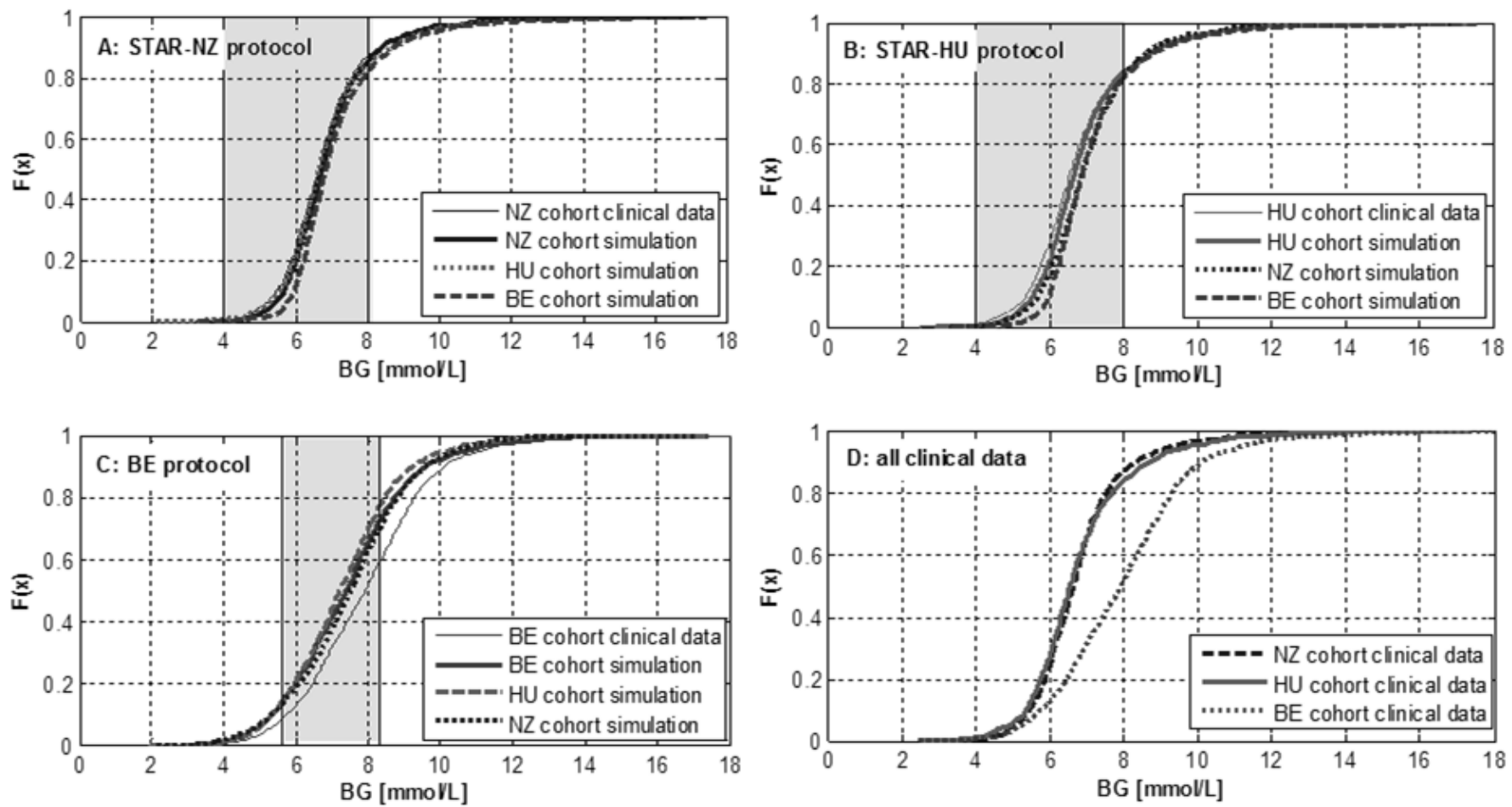

Fig. 6. Results of self- and cross- simulation: blood glucose (BG) CDFs for the clinical data and all 3 virtual cohort simulations (HU, NZ, BE). Shaded regions represent the glycaemic target band for that protocol for reference. Panel a) is the NZ protocol (see Table 3); b) is the HU protocol (see Table 4); and c) is the BE protocol (see Table 3); and d) compares all clinical data for completeness. $\mathrm{F}(\mathrm{x})$ is the cumulative fraction of data less than or equal to the corresponding BG, as per standard CDF definition.

across each of the cohorts, The median, IQR, and $5^{\text {th }}-95^{\text {th }}$ percentile range indicate the potential variability of $\mathrm{SI}_{\mathrm{n}+1}$ for any given $\mathrm{SI}_{\mathrm{n}}$ value. While data scarcity above an SI of greater than $\sim 10^{-3} \mathrm{~L} / \mathrm{mU} / \mathrm{min}$ results in wide and variable bounds, what is interesting is that below this threshold each of the cohorts display similar behaviour, as shown in Figure 5d. Thus, while median SI may vary between cohorts, hour-to-hour variability across the common SI range is consistent.

Figures 4 and 5 thus suggest that patient cohorts may differ in the SI range in which they spend most of their time, and have different inter-patient metabolic variability. However, underlying intra-patient metabolic variability is much more consistent. The hour-to-hour intra-patient variation between cohorts is very similar despite differences between protocols and clinical implementation, different metabolic SI levels in Figure 4, and despite not matching the cohorts for diagnostic code or other factors. Consequently, hour-to-hour changes in SI are independent from protocol, and are instead patient-specific,

TABLE IV

HUNGARIAN PROTOCOL: CROSS SIMULATION RESULTS, CLINICAL DATA SHADED. RESULTS ARE FOR THE FULL (MAX 120 HRS) PATIENT EPISODE

\begin{tabular}{cccc}
\hline \hline Hungarian (HU) protocol & $\begin{array}{c}\text { Cross validation on } \\
\text { NZ cohort }\end{array}$ & HU - Clinical data & $\begin{array}{c}\text { Cross validation on BE } \\
\text { cohort }\end{array}$ \\
\hline BG measures / day & 13.0 & 12.8 & 14.7 \\
Mean time between measurements (hrs) & $2.0[1.0-3.0]$ & $1.7[1.0-2.9]$ & $2.0[1.0-2.0]$ \\
Median BG [IQR] (mmol/L) & $6.9[6.3-7.6]$ & $6.6[5.9-7.4]$ & $6.9[6.4-7.6]$ \\
Time in 4.4-8.0 mmol/L band (\%) & 81.5 & 81.0 & 81.4 \\
Median [IQR] exogenous insulin rate (U/hr) & $3.0[1.5-4.5]$ & $3.0[1.5-5.0]$ & $4.5[2.5-8.0]$ \\
\hline Per-Patient Median BG (mmol/L) & $6.8[6.6-7.1]$ & $6.5[6.3-6.9]$ & $6.9[6.6-7.2]$ \\
\hline \hline
\end{tabular}

TABLE V

BELGIAN PROTOCOL: CROSS SIMULATION RESULTS, CLINICAL DATA SHADED. RESULTS ARE FOR THE FULL (MAX 120 HRS) PATIENT EPISODE

\begin{tabular}{cccc}
\hline \hline Belgian (BE) protocol & $\begin{array}{c}\text { Cross validation on } \\
\text { New Zealand cohort }\end{array}$ & $\begin{array}{c}\text { Cross validation on } \\
\text { HU cohort }\end{array}$ & BE - Clinical data \\
\hline BG measures / day & 9.5 & 9.4 & 9.9 \\
Mean time between measurements (hrs) & $4.0[1.0-4.0]$ & $4.0[1.0-4.0]$ & $1.0[1.0-4.0]$ \\
Median BG [IQR] (mmol/L) & $7.6[6.5-8.5]$ & $7.3[6.4-8.2]$ & $7.4[6.3-8.5]$ \\
Time in 4.4-8.0 mmol/L band (\%) & 58.2 & 69.1 & 62.9 \\
Median [IQR] exogenous insulin rate (U/hr) & $2.0[0.5-3.5]$ & $3.0[1.5-3.5]$ & $3.0[2.0-5.5]$ \\
\hline Per-Patient Median BG (mmol/L) & $7.3[7.0-7.9]$ & $7.1[6.7-7.5]$ & $7.3[6.9-7.9]$ \\
\hline \hline
\end{tabular}



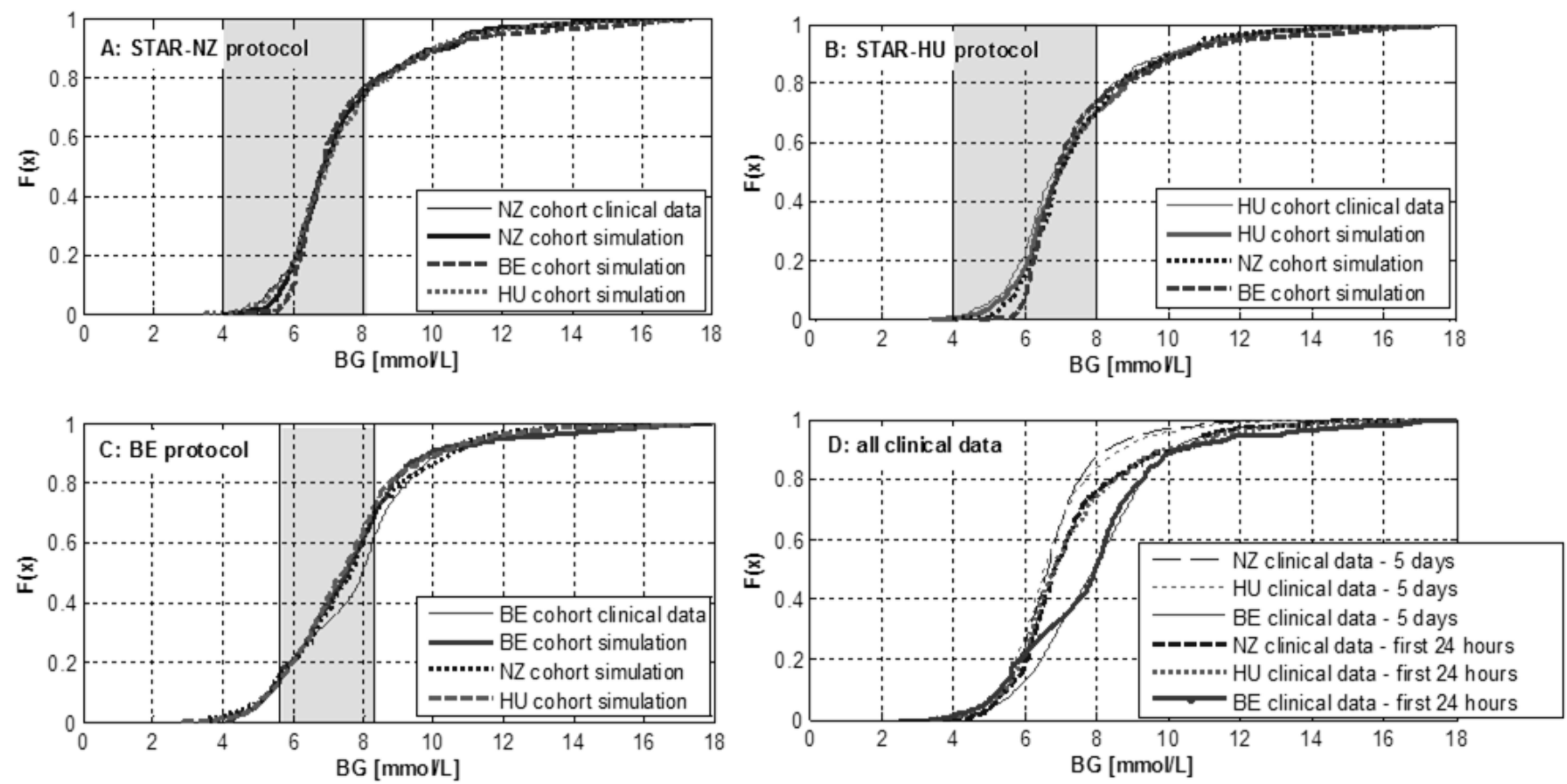

Fig. 7. Results of self- and cross- simulation for the first 24 hours only: blood glucose (BG) CDFs for the clinical data and all 3 virtual cohort simulations (HU, NZ, BE). Shaded green region represents the glycaemic target band for that protocol for reference. Panel a) is the NZ protocol (see Table 3); b) is the HU protocol (see Table 4); and c) is the BE protocol (see Table 3); and d) compares all clinical data for completeness. F(x) is the cumulative fraction of data less than or equal to the corresponding BG, as per standard CDF definition.

but similar across different cohorts. An important result is that, despite differences in the median cohort SI, stochastic forecasting models, such as those pictured in Figures $5 \mathrm{a}-\mathrm{c}$, are likely interchangeable and generalisable, potentially indicating a consistent physiological response.

\section{B. Protocol simulation using Virtual Trials}

Virtual trial results show the interaction of the model and model-based insulin sensitivity with glycaemic control protocols. Figure 6 and Tables 3 - 5 show the self- and crosssimulation results for each of the cohorts and protocols. Selfsimulation results show good matching in the STAR-NZ and STAR-Hu cohorts. Overall, self-validation results show that the ICING model can capture key BG dynamics, and reproduce clinical results in the case of protocolised insulin and nutrition treatments.

In the case of the BE cohort shown in Figure 6c, differences between the clinical and self-simulation results are attributed to clinical non-compliance, where $20-30 \%$ of interventions were modified slightly from protocol [40], combined with a lack of protocolisation around feed changes. The outcome is slightly higher clinical BG value than in the self- or cross- simulation results of Figure 6c.

Cross-simulation results show the interaction of SI and SI variability with a protocol on which the virtual patients were not treated. Cross-simulation result comparisons for all three protocols are similar, with median and IQR values falling within expected measurement error $(<5 \%)$. This results shows that the virtual trial model and methods are able to yield median (cohort and per-patient) and variability in predicted cohort BG outcomes that are very similar to the clinical data in all cases.

If only the first 24 hours of simulation are considered, cross simulation results show good matching above the $\sim 25^{\text {th }}$ percentile of BG outcomes for the STAR-HU and STAR-NZ protocols (Figures 7a and 7b), with similar modest deviation as in Figure 6 for the BE protocol (Figure 6c). Figure 8 shows that although by 24 hours cohort results begin to match well, during the first 20 hours BG outcomes are very cohort specific due to the interactions and differences in cohort starting BG and SI level. These starting differences between cohorts are most clearly seen in the ability for the protocols to reach lower extremes of glycaemic behaviour, where the results below the $25^{\text {th }}$ percentile in Figure $8 \mathrm{a}$ ) and b) tend to spread more.

\section{DISCUSSION}

Previous work has validated the virtual trial method in matched [35] cohorts, and extended the analysis to a New Zealand mixed-medical ICU cohort and a Belgian cardiovascular ICU cohort [37]. This work extends a virtual trial method to three unmatched medical ICU cohorts from three different countries and clinical practices, and examines the generalisability of virtual patient cohorts and the strengths and limitations of the virtual trial method. If virtual patient cohorts, created from clinical data, were interchangeable between ICUs, then it would be possible to significantly extend the use of virtual patients. This outcome would reduce the need for high quality clinical data and provide a common foundation glycaemic control design tool, similar to those used in Type 1 Diabetes research [51].

We hypothesised that virtual trials for protocol performance determination can be carried out on interchangeable, generalised cohorts. The results presented here show good matching between cross-simulated virtual trial simulations 

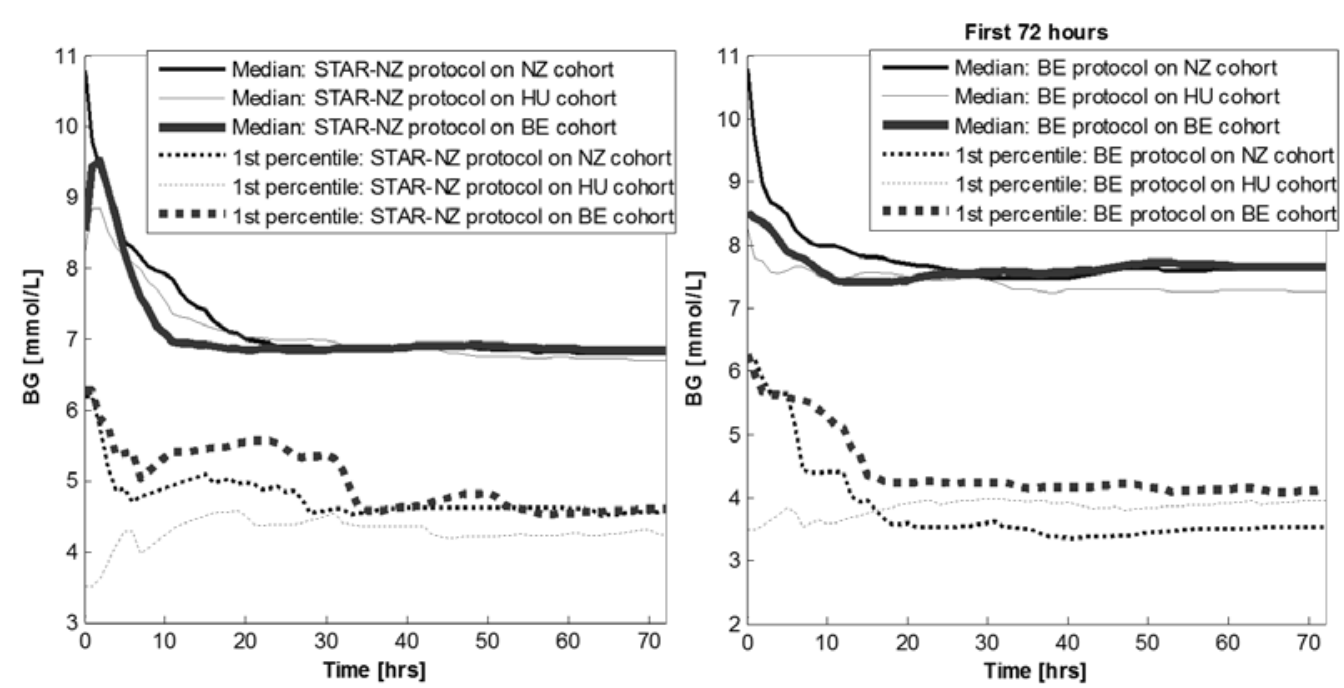

Fig. 8. First 72 hours of cumulative median BG data for the two most different protocols: a) STAR-NZ, and b) the BE titration protocol.

across a range of protocols and cohorts, as indicated by the overlap of the CDF's in Figure 6. These results suggest that overall performance of a glycaemic control protocol can be tested using virtual trials and a generalised cohort, without the need for local data collection.

A significant result of this work was the similarity across cohorts in terms of SI variability, despite differences in median cohort SI distributions. This result is similar to previous results in two different cohorts, using protocols that differ from those presented here, where SI variability was similar despite the fact that median SI was different between the cohorts [37]. This median SI and inter-patient variability likely differs between units based on the severity and type of illness or injury, and a potentially wide range of other cohort and demographic factors. In contrast, the similarity in SI variability strongly suggests that this hourly variability, driven by counter-regulatory and stress response physiology, may be more general than other factors in determining patient-specific metabolic behavior.

An added potential outcome of this study is that if a (larger) virtual patient cohort, sufficiently representative of intra-patient SI variability, could be created from multiple cooperating centres, there would not be a future need to build cohort specific forecasting models from retrospective data. The generality seen in Figure 5 indicates that this outcome could well be possible, although further work should look at specific sub-cohorts such as trauma or sepsis patients. Equally, arbitrary generalised virtual patients can be created based on clinically observed variability. While generalized population models go against the trend to make models more and more patient specific, these results show a good balance of exploiting generalisability to minimize clinical workload and data collection, while maintaining high safety and performance.

\section{A. Limitations on Virtual Trial Generalisability}

While this analysis suggests a good degree of generalisability in virtual trial cohorts, it is limited to 3 clinical cohorts. Further, this generalisability of virtual patient cohorts is subject to limitations and exclusion criteria based on protocol type and underlying assumptions.

All three cohorts were observed to have similar insulin sensitivity variability, but different cohort median SI. It is this similarity across intra-patient insulin sensitivity variability that determines the similarity in long term cohort results. Thus, for any cohort matched in intrapatient insulin sensitivity variability, similarity in 5 day virtual trial results is expected. This result reflects previous work using two clinically matched cohorts from a single intensive care unit, with very different insulin-nutrition treatments and glycaemic targets, but who were matched in underlying SI and SI variability [35]. Cohorts not matched in SI variability are likely to yield different glycaemic control results in

virtual trials.

Virtual trial results are not expected to be similar if protocols have limits on the maximum insulin dose much lower than the insulin dose at which insulin saturation effects ([52]) are observed. The protocol being tested must be flexible enough to allow a range of insulin doses, depending on underlying patient SI, whether it is being directly measured or not. For example, an insulin resistant patient will require higher insulin doses to achieve a target BG outcome than a more insulin sensitive patient. A strict sliding scale where insulin dose is directly proportional to BG will not likely have this flexibility, and highly resistant or highly fed patients will likely remain high in BG if insulin dose is very limited. On the other hand, sliding scale protocols where the change in insulin dose is proportional to $\mathrm{BG}$, such as the BE protocol in this paper or the protocols in the original validation paper [35], allow insulin doses to increase in patients with low SI.

A limitation of this method is that virtual trials are not expected to give similar results in patients where the starting BG and the time taken to reduce BG to the protocol median BG and/or target range are very different between cohorts, especially over the first 24-48 hours. These early outcomes are heavily influenced by patient and cohort SI and nutritional delivery. While in these results the 24 hour BG outcome results were also very similar, the 12 hour results are very different (Figure 8) as the time taken for each cohort to be lowered into the target range differs based on starting BG and cohort median insulin sensitivity. The shorter the time to lower BG into the target range relative to the length of each virtual patient, the closer virtual trial BG outcomes will be in cohorts with different median SI or feed rates.

In addition, over the first 24 hours the greatest variability in low blood glucose outcomes was observed between cohorts. This outcome suggests that when designing and testing protocols for use in a different cohort, virtual trials with interchanged cohorts may not fully or accurately reflect safety and performance over the first 12-24 hours, which can be important to outcome [53]. In addition, in terms of clinical compliance and equipoise over the adoption or use of a new protocol, these first hours can be crucial to overall protocol 
performance and uptake. For this reason, it may be wise to simulate potential BG outcomes in a cohort of 'difficult' patients as well as a generalised cohort, to determine a worst case protocol design outcome for protocol results over the first 24 hours.

Finally, generalised virtual trial methods are not able to give estimations of total insulin or nutrition delivery, as this delivery is cohort specific and will depend on underlying condition and SI, and clinical preferences in nutrition type and range. While protocol performance is generally of more concern than its consumables, clinical preference and goals for total feed delivery may be an obstacle for protocol implementation where a glycaemic control protocol modulates both glucose and insulin. Results around total insulin and nutrition delivery from generalised virtual trials should be treated with caution where the underlying SI from the targeted cohort is unknown.

\section{B. Study Limitations and Future Work}

This study utilized the ICING model. This model, as with all models, has underlying assumptions and has regions for BG and insulin-nutrition interventions in which it is most accurate. In this case, many model parameters are fixed as they are not currently identifiable using available bedside data. As a result, the SI parameter reflects both peripheral insulin sensitivity and patient-specific deviation from population-based model parameters, all of which could be time-varying. It thus lumps changes in patient response into the SI value. However, the fact that SI has been validated in its effective clinical use for glycaemic control, and can be used in virtual trials to accurately simulate clinical outcomes [35, 41], suggests that the model overall captures key dynamics and dynamic shapes. This model thus balances the need for practical bedside identifiability [54] and use with model accuracy to underlying physiology [54, 55]

A primary limitation of this study is the use of only 3 cohorts, and only 2 glycaemic control frameworks, from intensive care units that are primarily western in ethnic groups and clinical practice. The HU cohort had much higher APACHE II score than the NZ cohort, indicating differences in illness severity, but this score was not available for the BE cohort. These cohorts were drawn from different countries and clinical practices, and had different underlying SI. In future, these results should be tested on patient data cohorts from potential different ethic cohorts, such as those from Asian or Asia-Pacific intensive care units. Should SI variability prove to be similar across a wider range of ethnic origins, this work would provide a strong case for generalisable virtual trials to give a reasonable overview of likely BG outcomes.

The use of unmatched cohorts was chosen by design to test the generalisability of the virtual patient methods tested and to help find the underlying reasons for successful validation. However, clinically matched cohorts would provide a stronger validation result as in [35], even if limited in application since local patient data would be required to create a local virtual cohort based on the evidence of results using matched cohorts. In this case, the cohorts are similar in age and do not have diagnosed diabetes patients, but are otherwise general.

Two of the protocols used in this analysis, STAR-NZ and STAR-HU, were similar in design dynamic, if not in clinical use and delivery. These similarities and differences give an indication of how very different clinical implementation conditions and nutritional delivery can impact virtual trial results. In this case, STAR-NZ and STAR-HU are able to give similar clinical results despite differences in insulin delivery and nutrition rate.

It is very difficult to completely or perfectly validate these results or any in silico virtual trial. An ideal real clinical trial would treat a patient, and then take them back in time to treat them differently, all else the same. In theory these results could be fully validated implementing all protocols clinically in each unit, preferably with strict compliance and matched cohorts. This outcome could only be achieved prospectively at some cost, and was not possible here. However, the results presented strongly suggest that relatively small 20 patient virtual trial cohorts can capture a majority of cohort outcomes, and that larger cohorts might do as well or better.

\section{CONCLUSIONS}

Virtual patients were generated from clinical data from three different intensive care units using a glucose-insulin pharmacokinetic model. These virtual patients were used in virtual trials to test the generalisability of virtual patients and virtual trial methods across different ICUs. It was found that although cohort median insulin sensitivity was different between ICUs, hour-to-hour variability in this insulin sensitivity was similar. As a result, different virtual patient cohorts were able to closely replicate glycaemic control outcomes in virtual trials across different protocols. These results provide evidence for the generalisability of virtual patient cohorts and the virtual patient method to assess likely long-term glycaemic control outcomes during protocol design and testing. While generalisable, this virtual patient method has several important limitations, in that it may not accurately reflect cohort behaviour in the first 24 hours in some cohorts, and protocols tested must be sufficiently flexible to adapt to more resistant or highly fed patients. Overall, this paper presents results supporting a generalised virtual trial method, and discusses the strengths and limitations of this method.

\section{REFERENCES}

1. McCowen, K.C., A. Malhotra, and B.R. Bistrian, Stress-induced hyperglycemia. Crit Care Clin, 2001. 17(1): p. 107-124.

2. Marik, P.E. and R. Bellomo, Stress hyperglycemia: an essential survival response! Crit Care, 2013. 17(2): p. 305.

3. Dungan, K.M., S.S. Braithwaite, and J.C. Preiser, Stress hyperglycaemia. Lancet, 2009. 373(9677): p. 1798-807.

4. $\quad$ Egi, M., S. Finfer, and R. Bellomo, Glycemic control in the ICU. Chest, 2011. 140(1): p. 212-20.

5. $\quad$ Egi, M., et al., Variability of blood glucose concentration and shortterm mortality in critically ill patients. Anesthesiology, 2006. 105(2): p. 244-52.

6. $\quad$ Egi, M., et al., Hypoglycemia and outcome in critically ill patients. Mayo Clin Proc, 2010. 85(3): p. 217-24.

7. Krinsley, J.S., Association between hyperglycemia and increased hospital mortality in a heterogeneous population of critically ill patients. Mayo Clin Proc, 2003. 78(12): p. 1471-1478.

8. $\quad$ Bagshaw, S.M., et al., The impact of early hypoglycemia and blood glucose variability on outcome in critical illness. Crit Care, 2009. 13(3): p. R91.

9. Krinsley, J., et al., Mild hypoglycemia is strongly associated with increased intensive care unit length of stay. Ann Intensive Care, 2011. 1: p. 49. 
10. Krinsley, J.S. and J.C. Preiser, Time in blood glucose range 70 to $140 \mathrm{mg} / \mathrm{dl}>80 \%$ is strongly associated with increased survival in non-diabetic critically ill adults. Crit Care, 2015. 19: p. 179.

11. Finfer, S., et al., Hypoglycemia and risk of death in critically ill patients. N Engl J Med, 2012. 367(12): p. 1108-18.

12. Van den Berghe, G., et al., Intensive insulin therapy in the critically ill patients. N Engl J Med, 2001. 345(19): p. 1359-1367.

13. Van den Berghe, G., et al., Intensive insulin therapy in the medical ICU. N Engl J Med, 2006. 354(5): p. 449-61.

14. Krinsley, J.S., Effect of an intensive glucose management protocol on the mortality of critically ill adult patients. Mayo Clin Proc, 2004. 79(8): p. 992-1000.

15. Lin, J., et al., Stochastic modelling of insulin sensitivity and adaptive glycemic control for critical care. Computer Methods and Programs in Biomedicine, 2008. 89(2): p. 141-152.

16. Finney, S.J., et al., Glucose control and mortality in critically ill patients. Jama, 2003. 290(15): p. 2041-2047.

17. Chase, J.G., et al., Organ failure and tight glycemic control in the SPRINT study. Crit Care, 2010. 14(4): p. R154.

18. Signal, M., et al., Glycemic levels in critically ill patients: are normoglycemia and low variability associated with improved outcomes? J Diabetes Sci Technol, 2012. 6(5): p. 1030-7.

19. Penning, S., et al., Glucose control positively influences patient outcome: A retrospective study. J Crit Care, 2015. 30(3): p. 455-9.

20. Van den Berghe, G., et al., Outcome benefit of intensive insulin therapy in the critically ill: Insulin dose versus glycemic control. Crit Care Med, 2003. 31(2): p. 359-366.

21. Finfer, S., et al., Intensive versus conventional glucose control in critically ill patients. N Engl J Med, 2009. 360(13): p. 1283-97. Brunkhorst, F.M., et al., Intensive insulin therapy and pentastarch resuscitation in severe sepsis. N Engl J Med, 2008. 358(2): p. 12539.

Preiser, J.C., et al., A prospective randomised multi-centre controlled trial on tight glucose control by intensive insulin therapy in adult intensive care units: the Glucontrol study. Intensive Care Med, 2009. 35(10): p. 1738-48.

Pretty, C.G., et al., Variability of insulin sensitivity during the first 4 days of critical illness: implications for tight glycemic control. Ann Intensive Care, 2012. 2(1): p. 17.

Langouche, L., et al., Effect of intensive insulin therapy on insulin sensitivity in the critically ill. J Clin Endocrinol Metab, 2007. 92(10): p. 3890-7.

Dickson, J.L., C.A. Gunn, and J.G. Chase, Humans are horribly variable. International Journal of Clinical and Medical Imaging, 2014. 1(2).

Chase, J.G., et al., Tight glycemic control in critical care-The leading role of insulin sensitivity and patient variability: A review and model-based analysis. Computer Methods and Programs in Biomedicine, 2011. 102(2): p. 156-171.

28. Van Herpe, T., et al., LOGIC-Insulin Algorithm-Guided Versus Nurse-Directed Blood Glucose Control During Critical Illness: The LOGIC-1 single-center randomized, controlled clinical trial. Diabetes Care, 2013. 36(2): p. 189-194.

29. Blaha, J., et al., Intensive insulin therapy in critically ill patients: comparison of standard and MPC protocols. Intensive Care Med, 2005. 31(S1): p. S203.

30. Blaha, J., et al., Comparison of three protocols for tight glycemic control in cardiac surgery patients. Diabetes Care, 2009. 32(5): p. 757-61.

31. Plank, J., et al., Multicentric, randomized, controlled trial to evaluate blood glucose control by the model predictive control algorithm versus routine glucose management protocols in intensive care unit patients. Diabetes Care, 2006. 29(2): p. 271-6.

32. Evans, A., et al., Pilot proof of concept clinical trials of Stochastic Targeted (STAR) glycemic control. Annals of Intensive Care, 2011. 1(1): p. 38.

33. Stewart, K., et al., Safety, Efficacy and Clinical Generalization of the STAR Protocol: A Retrospective Analysis. Annals of Intensive Care, 2016. in-press.

34. Lonergan, T., et al., A simple insulin-nutrition protocol for tight glycemic control in critical illness: development and protocol comparison. Diabetes Technol Ther, 2006. 8(2): p. 191-206.

35.
36.

.

38.

40.

41.

42.

43.

44.

45.

46.

47.

48.

49.

Wilinska, M.E., L. Chassin, and R. Hovorka, In Silico TestingImpact on the Progress of the Closed Loop Insulin Infusion for
Critically Ill Patients Project. Journal of Diabetes Science and Technology, 2008. 2(3): p. 417-423.

Suhaimi, F., et al., What Makes Tight Glycemic Control (TGC) Tight? The impact of variability and nutrition in 2 clinical studies. Journal of Diabetes Science and Technology, 2010. 4(2): p. 284298.

Lin, J., et al., Stochastic modelling of insulin sensitivity variability in critical care. Biomedical Signal Processing and Control, 2006.

$$
\text { 1(3): p. 229-242. }
$$

glycemic control for critical care. Comput Methods Prograve Biomed, 2008. 89(2): p. 141-52.

Penning, S., et al., In silico assessment of a computerized modelbased glycaemic control approach in a Belgian medical intensive care unit, in 19th IFAC World Congress2014, IFAC (PapersOnline): Cape Town, South Africa. p. 6-pages.

Fisk, L., et al., STAR Development and Protocol Comparison. IEEE Trans Biomed Eng, 2012. 59(12): p. 3357-3364.

Singer, P., et al., ESPEN Guidelines on Parenteral Nutrition: intensive care. Clin Nutr, 2009. 28(4): p. 387-400.

Cerra, F.B., et al., Applied nutrition in ICU patients. A consensus statement of the American College of Chest Physicians. Chest, 1997. 111(3): p. 769-78.

Lin, J., et al., A physiological Intensive Control Insulin-NutritionGlucose (ICING) model validated in critically ill patients. Comput

Methods Programs Biomed, 2011. 102(2): p. 192-205.
Stewart, K.W., et al., Stochastic Model Predictive (STOMP) glycaemic control for the intensive care unit: Development and virtual trial validation. Biomedical Signal Processing and Control, 2015. 16: p. 61-67.

Pretty, C.G., et al., Interstitial insulin kinetic parameters for a 2compartment insulin model with saturable clearance. Computer methods and programs in biomedicine, 2014. 114(3): p. e39-45.

Chase, J.G., et al., Targeted glycemic reduction in critical care using closed-loop control. Diabetes Technol Ther, 2005. 7(2): p. 274-82. Hann, C.E., et al., Integral-based parameter identification for longterm dynamic verification of a glucose-insulin system model. Comput Methods Programs Biomed, 2005. 77(3): p. 259-270.

Pretty, C.G., Analysis, classification and management of insulin sensitivity variability in a glucose-insulin system model for critical illness, in Mechanical Engineering2012, University of Canterbury: Christchurch, New Zealand. p. 169.

50. Docherty, P.D., J.G. Chase, and T. David, Characterisation of the iterative integral parameter identification method. Medical and Biological Engineering and Computing, 2012: p. 1-8.

51. Visentin, R., et al., The university of Virginia/Padova type 1 diabetes simulator matches the glucose traces of a clinical trial. Diabetes technology \& therapeutics, 2014. 16(7): p. 428-34.

52. Chase, J.G., et al., A glucose-insulin pharmacodynamic surface modeling validation and comparison of metabolic system models. Biomed Signal Proces, 2009. 4(4): p. 355-363.

53. Bagshaw, S., et al., The impact of early hypoglycemia and blood glucose variability on outcome in critical illness. Critical Care, 2009. 13(3): p. R91.

54. Docherty, P.D., et al., A graphical method for practical and informative identifiability analyses of physiological models: a case study of insulin kinetics and sensitivity. Biomed Eng Online, 2011. 10: p. 39.

55. Chase, J.G., et al., Model-based glycaemic control in critical care a review of the state of the possible. Biomedical Signal Processing and Control, 2006. 1(1): p. 3-21. 